in women has led to ignorance of or disbelief in its existence, particularly by gynaecologists and obstetricians, most of whom do not number the microscope among their favourite instruments.

That the disease is a force to be reckoned with is becoming abundantly clear if only on account of the increasing numbers of cases. When it was first included as a separate item in the returns from the venereal diseases clinics of England and Wales in 1954 under the heading of "non-gonococcal urethritis" in males only the number of cases was 10,794. In 1972 the number of cases in men was 65,895- as compared with 54,974 cases of gonorrhoea in men, women, and children. A further increase occurred in 1973, though exact figures are not yet available.

Fox has recently described ${ }^{1}$ a study conducted in a general practice with a mainly working-class clientele in London. He investigated 325 women of whom $80 \%$ were in the third or fourth decade of life and of whom $95 \%$ were married. Ninetyeight were examined because they complained of urogenital symptoms; 227 had no symptoms and were examined in the course of routine postnatal care, for contraception, or for cervical cytological tests. Patients were excluded if they or their consorts had received systemic antibiotics within the preceding three weeks or if they were pregnant. Non-specific genital infection was diagnosed in the women if specific pathogens were absent and microscopy showed 50 or more polymorphonuclear leucocytes in several high-power fields of the cervical secretion and if this finding was confirmed by cervical cytological test. This diagnosis was made irrespective of the presence or absence of clinical evidence of cervicitis. Several patients also had microscopical evidence of urethritis. Early morning specimens of urine were obtained from consorts who had been asked to take no fluids overnight and were examined microscopically after centrifugation; if 10 or more polymorphonuclear leucocytes were seen in several high-power fields a diagnosis of urethritis was made. In all, 182 women were found to have non-specific genital infection and 54 $(30 \%)$ of their consorts had evidence of infection. Of the consorts of 113 women without evidence of such infection only two $(1 \cdot 8 \%)$ had evidence of the condition. Almost all $(89 \%)$ of the 54 infected men were asymptomatic and had no previous history of urethritis. Many more of the couples in whom only one partner was affected apparently used condoms regularly than did those who shared infection, suggesting that the use of condoms might be an effective barrier to transmission of infection.

There are, thus, sound reasons for believing that nonspecific genital infection is quite common in general practice, even though most infections do not present in easily recognizable form. Some general practitioners refer men with urethral discharge straight to a clinic, but many patients prefer to stay with their own doctor and many doctors believe it is right that they should do so. The method which some adopt is to give an injection of penicillin at once and refer the patient to a clinic only if his symptoms are not relieved. This may be damaging to the individual, to his sexual partner, and to the public health, and it also adds to the burdens of the venereologist. Proper care of these patients should mean accurate diagnosis, adequate treatment, satisfactory tests for cure, and the tracing of contacts, including sources of infection and those whom the patient may have infected. Accurate diagnosis requires careful clinical examination and the help of a laboratory. Treatment is not always easy. The condition does not respond to penicillin; the most effective remedies seem to be tetracycline or oxytetracycline, $250 \mathrm{mg}$ by mouth every 6 hours for one to three weeks. There is some evidence that the longer duration of treatment may give better results, ${ }^{2}$ but little to support the view that doubling the dose is any help. During treatment the patient should abstain from alcohol and sexual intercourse. ${ }^{3}$ Because this condition is sometimes prone to relapse observation should, ideally, extend over three months, but it may be difficult to persuade patients to abstain from intercourse for so long. During observation the patient, if a male, should be seen on at least two occasions in the early morning before the overnight urine has been passed for intrameatal smears, inspection of the urine, and microscopy of the urinary sediment. It is wise also to perform at least one microscopical examination of the prostatic fluid, for nonspecific infection is the commonest cause of chronic prostatitis, often asymptomatic. Wives and sexual partners should always be examined for the disease and treated if necessary.

The management of these cases requires a good deal of work for the practitioner but is well within his compass if the case remains straightforward and he has access to laboratory help. The alarming increase in numbers of cases may make it essential that he should bear part of the burden.

\footnotetext{
1 Fox, H., British Fournal of Venereal Diseases, 1974, 50, 125.

2 John, J., British fournal of Venereal Diseases, 1971, 47, 266.
}

\section{Cardiovascular Disease and Peptic Ulcer}

The possibility that disease in blood vessels may play a part in the aetiology of peptic ulceration has long been considered. Mucosal necrosis and ulceration have been ascribed to vascular disease in the gut wall or to submucosal haemorrhage. But in recent years some associations have been described between vascular disease and peptic ulcer or gastrointestinal bleeding which at first sight seem inexplicable or even incompatible with each other.

Patients with coronary artery disease have a somewhat greater than average frequency of peptic ulcer, particularly duodenal ulcer. $^{12}$ A post-mortem survey showed a similar association between abdominal aortic aneurysms and peptic, especially duodenal, ulcers. ${ }^{3}$ Further, ischaemic heart disease was found to be extremely common in those cases. Finally, gastric ulceration has been found especially frequently in patients with radiological evidence of abdominal aortic calcification. ${ }^{4}$ But there are problems in determining whether these associations are real. Peptic ulceration and vascular disease are both common, and it is difficult to produce unbiased and reliable data giving exact levels of frequency. Both go through various grades from minimal to gross, and the decision when a vascular change becomes a disease in Western man is a matter of arbitrary judgement, for some degree of abnormality is general in adult life. Likewise the frequency with which peptic ulcer is found will depend partly on the enthusiasm of the searcher, and this can be particularly obvious where post-mortem assessment of scarring due to a healed ulcer is concerned.

If a general association between vascular disease and peptic ulcer is accepted, the basis for it is hard to define. Possible and potentially interrelated factors include a common association with smoking, a common relationship with social class, whatever that in turn might imply, and simple damage of the mucosa by a poor blood supply. 
The association of coronary artery disease with smoking is well known. Peptic ulcer is commoner in smokers than in non-smokers, and smokers without an ulcer have an increased liability to its subsequent development. ${ }^{5-7}$ Ulcer morbidity and mortality vary with socioeconomic status, and, at least in Great Britain, both seem to be higher in people who are relatively impoverished. ${ }^{8}{ }^{9}$ This trend is the reverse of what is popularly accepted-the hard-working executive with an ulcer-but that picture is probably based on figures published 30 years ago showing that mortality from duodenal ulcer was then distinctly greater in social class I than in any other. ${ }^{10}$ The reason for the change is unknown, but now the relationship between mortality and social class seems to be the same for gastric and duodenal ulcer and for coronary artery disease. ${ }^{9}$

Two associations which are hard to understand include an inverse correlation between blood pressure and frequency of peptic ulcer, and an association between idiopathic gastrointestinal bleeding and aortic stenosis. The former was detected during a survey conducted in Israel, when the incidence of peptic ulcer was found to be somewhat diminished in men with relatively low diastolic blood pressures. ${ }^{11}$ The results are the same as those obtained over 40 years ago in a study conducted by Glass. ${ }^{12}$ No logical explanation seems possible. The second association, between gastrointestinal bleeding and aortic stenosis, ${ }^{13}$ may be due to ischaemic mucosal necrosis as a result of poor gastrointestinal perfusion. But, if this is so, it seems somewhat surprising that gastrointestinal bleeding is not reported more frequently as a complication of hypotensive cardiac infarction, when the insult might be expected to be more severe, even if brief.

1 Watkinson, G., Gastroenterologia, 1958, 89, 292.

2 Brooks, F. P., et al., American fournal of Medical Science, 1963, 245, 277.

3 Jones, A. W., et al., Gut, 1970, 11, 679.

4 Elkeles, A., American fournal of Roentgenology, Radium Therapy, and Nuclear Medicine, 1964, 91, 744 .

Doll, R., et al., Lancet, 1958, 1, 657.

- Monson, R. R., Gastroenterology, 1970, 58, 337

Doll, R., and Hill, A. B., British Medical fournal, 1964, 1, 1399.

${ }^{8}$ Litton, A., and Murdoch, W. R., Gut, 1963, 4, 360.

Registrar General's Decennial Supplement on Occupational Mortality for 1961 for England and Wales. London, H.M.S.O., 1971.

10 Morris, J. N., and Titmuss, R. M., Lancet, 1944, 2, 841.

11 Medalie, J. H., et al., Lancet, 1970, 2, 1225.

12 Glass, G., and Landau, A., Archives des Maladies de l'Appareil Digestif, $1929,19,948$.

13 Cody, M. C., et al., American Fournal of Digestive Diseases, 1974, 19, 393.

\section{Lead and Intelligence}

Lead in ever increasing amounts is being mined and refined and widely distributed in the environment. In certain occupations and domestic situations exposure to it can cause well recognized clinical effects. In the case of children it is accepted that acute poisoning may sometimes result in irreversible brain damage. The problem that continues to preoccupy many people is whether an exposure to lead that does not cause recognized poisoning can nevertheless cause subclinical lead poisoning.

This fear is often linked with the widespread use of lead in petrol and its subsequent distribution from motor-car exhausts. Thus a special study was set up to look at the exposure of people living near Gravelly Hill-“"Spaghetti Junction"-and a first report has been issued." This provided no evidence of overexposure to lead, but more work is planned.

The problem remains-what is "overexposure" to lead? A recent book entitled Subclinical Lead Poisoning ${ }^{2}$ might be expected to provide some of the answers, but unfortunately it hardly discusses this particular problem at all. The ques- tion was discussed at greater length at a conference on low level lead toxicity held in the U.S.A., and the 32 papers have recently been published. ${ }^{3}$ Five of the contributions reported studies on the intelligence and behaviour of children. In one of these 370 children were divided into five groups according to evidence of their previous exposure to lead and assessed by psychological tests and school performance. As expected, some of the children with a history of clinical lead poisoning showed seriously impaired performance. Harmful effects were noted in some children with high blood levels of lead but no clinical history of poisoning when compared with children with normal blood levels of lead, including some with high tooth levels of lead suggesting heavy exposure in the past.

A study of 700 children ${ }^{5}$ with "low average mental abilities" disclosed 58 with an increased body burden of lead, and about a quarter of these had some minor neurological dysfunction. The problem of whether a low mental ability is caused by the exposure to lead or itself results in the heavier exposure of small children to lead in ambient dirt is further exemplified by the problem of hyperactivity in children. O. J. David, ${ }^{6}$ supporting his thesis that exposure to lead is a cause of hyperactivity, admitted that the number of children falling into a group in which lead was the "highly probable cause" was too small to be conclusive evidence of the association.

In a study in London ${ }^{7}$ there were on average higher blood levels of lead in children and their mothers living near a lead factory than in those whose homes were a short distance further away, but no differences could be detected in the intelligence and behaviour of the schoolchildren from these two groups. Steps were taken to trace children who had lived in the area of higher exposure up to the age of 2 years. These also were normal, which suggests that any ill effect such an exposure had had in infancy was reversible.

While there is nothing in all this work to cause alarm it is clear that better evidence is needed before anyone can state with confidence the degree of exposure to lead which carries no risk to a child's brain. A clear assessment of small difference in mental ability and behaviour between groups of children will be difficult for a long time yet, but much more is now known about likely sources of exposure. While air levels of lead are convenient for assessing a general environmental level of exposure, this is not the route by which dangerous amounts of lead are absorbed in the domestic environment. ${ }^{8}$ Painstaking studies ${ }^{910}$ with stable lead isotopes, feasible only on consenting adults, have shown that only $15 \mu \mathrm{g}$ of lead is absorbed each day from the atmosphere. The presence of lead at high levels in the soil from old lead mine workings need not result in children living near them being exposed to excessive levels. ${ }^{11}$ Lead in ingested dirt, usually containing fragments of old lead-containing paint, is the commonest source of exposure in children in addition to the 200-300 $\mu \mathrm{g}$ present in a normal daily diet. ${ }^{12}$ But the exposure of each child even in a single household may differ widely in accordance with his age, habits, and behaviour. Since it is unlikely that many opportunities will arise for the assessment of young children's intelligence and behaviour before they are exposed to lead in their domestic environment, the problem of whether a high body burden of lead causes or was caused by defects in mental ability will long remain unsolved.

Every effort must be made to reduce domestic sources of lead, and there is no excuse for permitting factories in builtup areas to continue to discharge wastes that will raise the lead levels of dust in near-by homes. The lead levels of the soils in which our homes are built are not a matter of con- 